\title{
PARALLEL UMBILICAL HYPERSURFACES
}

CURTIS M. FULTON

The only umbilical surfaces of Euclidean space are planes and spheres. We propose to study umbilical hypersurfaces in Riemannian space with the added condition that they constitute a system of parallels. As will be shown, such families only exist in spaces of constant curvature.

We will adhere throughout to the spirit of Weatherburn's notation [3]. Greek indices take the values $1,2, \cdots, m=n+1$; the range of Latin indices is $1, \cdots, n$.

Consider the equations $y^{\alpha}=y^{\alpha}\left(x^{1}, x^{2}, \cdots, x^{n}, s\right)$. For each value of $s$ we have a hypersurface and if the $x$ 's are fixed, the equations represent a trajectory of the family of hypersurfaces. Since we want a system of parallels [3, pp. 80-82], the trajectories used are geodesics orthogonal to the hypersurfaces. Making $s$ the arc length $\partial_{s} y^{\alpha}=N^{\alpha}$ is the unit normal and for the intrinsic derivative of $y^{\alpha}{ }_{, i}$ we find

$$
D_{s} y^{\alpha}, i=N_{; i}^{\alpha},
$$

where $N^{\alpha} ;$ is the tensor derivative of $N^{\alpha}\left[3, \mathrm{p}\right.$. 135]. Also, $D_{8} N^{\alpha}=0$. If we eliminate the $x$ 's and solve for $s$ we get the representation $U\left(y^{1}, y^{2}, \cdots, y^{m}\right)=s$ for the family of hypersurfaces. Because of $U_{, \alpha} N^{\alpha}=1, U_{, \alpha}=N_{\alpha}$. Moreover, the hypersurfaces are required to be totally umbilical which means $\Omega_{i j}=-L g_{i j}[3$, p. 139] and this in turn leads to $[3$, p. 136]

$$
N^{\alpha}{ }_{i i}=L y^{\alpha}{ }_{, i} .
$$

THEOREM. If through every point in all directions there exist hypersurfaces belonging to a system of parallel umbilical hypersurfaces, then the space has constant Riemannian curvature $K, L_{, i}=0,-d_{s} L=L^{2}+K$, and conversely.

If we take $U=s$ as the equation of the family of hypersurfaces $N$ may be regarded as a function of the $y$ 's and we write (2) in the form $\left(N_{\alpha, \beta}-L a_{\alpha \beta}\right) y^{\beta}{ }_{, i}=0$. Furthermore, $N_{\alpha, \beta}=U_{, \alpha \beta}=N_{\beta, \alpha}$. We now think of $N_{\alpha, \beta}-L a_{\alpha \beta}$ expressed in terms of $N$ and an orthogonal ennuple [1, pp. 96-97] consisting of vectors perpendicular to $N$. The tensor in question is seen to be $N_{\alpha} N_{\beta}$ times a scalar factor. Multiplication by $N^{\alpha}$ shows that

Received by the editors July 12, 1964. 


$$
N_{\alpha, \beta}=L\left(a_{\alpha \beta}-N_{\alpha} N_{\beta}\right) .
$$

Conversely, (2) follows from (3) and (3) also implies that $N$ is a gradient $[3$, p. 60$]$ and this latter fact tells us that we are dealing with a system of parallels [3, p. 82]. In other words (3) is characteristic for parallel umbilical hypersurfaces.

The system of equations (3) has been shown to be completely integrable [2] if the Riemannian curvature $K$ is constant and $-L_{, \alpha}$ $=\left(L^{2}+K\right) N_{\alpha}$. These conditions were also proven necessary for the existence of solutions of (3) at all points in arbitrary directions. Clearly, the equations for $L$ are equivalent to $L_{, i}=0$ and $-d_{8} L$ $=L^{2}+K$.

We note that $L$ is constant on a hypersurface and for an individual hypersurface we have the constant curvature $[1, \mathrm{p} .185]$

$$
K(s)=L^{2}+K \text {. }
$$

THEOREM. If, in a space of constant $K$, one hypersurface in a family of parallels is umbilical, all are.

For the proof we use an identity which can be derived directly taking into account the definition of the curvature tensor [3, p. 110] and $D_{s} N^{\alpha}=0$, namely $D_{s} N^{\alpha}{ }_{; i}=R^{\alpha}{ }_{\beta \lambda \zeta} N^{\beta} N^{\lambda} y^{\zeta}, i$. It follows that in our case $D_{s} N^{\alpha}{ }_{; i}=-K y^{\alpha}{ }_{, i}$. We assume that $N^{\alpha}{ }_{i i}=L_{0} y^{\alpha}{ }_{, i}$ for the hypersurface $s=0$. Let $L$ be the solution of the differential equation $-d_{s} L=L^{2}+K$ which takes the value $L_{0}$ when $s=0$. For a fixed $i$ we set $w^{\alpha}=N^{\alpha}{ }_{; i}-L y^{\alpha}{ }_{, i}$ and conclude with the aid of (1) that $w^{\alpha}$ satisfies the differential equation $D_{s} w^{\alpha}=-L w^{\alpha}$. Now as $s=0, w^{\alpha}=0$ and so $w^{\alpha} \equiv 0$ are the unique solutions of the differential equations which proves our theorem.

In spaces of constant curvature then, any umbilical hypersurface gives rise to a family of parallel umbilical hypersurfaces. For convenience we use the latter concept to investigate the totality of umbilical hypersurfaces. We restrict our discussion to hyperbolic space which offers the greatest variety. Returning to the differential equation $-d_{8} L=L^{2}+K$, we observe that its solutions naturally fall into three categories.

(I) $L^{2}+K<0$. Denoting the square root of $-K$ by $k$, we get solutions containing an arbitrary constant $C$ in the form $L=k \tanh k(s-C)$. Hence for $s=C, L=0$ which corresponds to a hyperplane [3, p. 139] and other values of $s$ belong to equidistant hypersurfaces.

(II) $L^{2}+K>0$. Here $L=k$ coth $k(s-C)$. If, by definition, $z^{\alpha}=y^{\alpha}{ }_{i} \operatorname{csch} k(s-C)$ it is found that $D_{s} z^{\alpha}=0$. Thus $z^{\alpha}$ has constant magnitude and that of $y^{\alpha}{ }_{, i}$ involves the factor $\sinh k(s-C)$. We infer 
that for $s=C, y^{\alpha}=$ const, $L$ being undefined. Hence in case (II) we are dealing with hyperspheres.

(III) $L^{2}+K=0$. In this case it has been demonstrated [2] that the vectors $N$ and hence the geodesic trajectories are all parallel. Consequently the family consists of limiting hypersurfaces or hyperhorospheres. They all have curvature zero.

Elliptic space admits of case (II) only, but the hyperspheres are geometrically the same as equidistant hypersurfaces.

\section{REFERENCES} 1949.

1. L. P. Eisenhart, Riemannian geometry, Princeton Univ. Press, Princeton, N. J.,

2. C. M. Fulton, Parallel vector fields, Proc. Amer. Math. Soc. 16 (1965), 136-137.

3. C. E. Weatherburn, An introduction to Riemannian geometry and the tensor calculus, University Press, Lambridge, 1957.

University of California, Davis

\section{THE RING $C(X)$ DETERMINES THE CATEGORY OF $X$}

W. W. COMFORT AND STELIOS NEGREPONTIS ${ }^{1}$

Summary and introduction. Our point of departure is the assertion (justified in Lemma 1.2) that certain topological properties of an arbitrary space $Y$ are inherited by each subset of $Y$ which meets each nonempty $G_{\delta}$ subset of $Y$. We consider specifically only the properties "is of second category" and "is a Baire space," but other properties in the same vein will occur readily to the reader. Specializing quickly to completely regular Hausdorff spaces, we recall that $X$ is $G_{\delta}$-dense (in the sense just described) in its Hewitt completion (= "realcompactification") $v X$, so that $X$ possesses each of the properties in question if and only if $v X$ does. This means that algebraic properties of the ring $C(X)$ of real-valued continuous functions on $X$ determine, for example, the category of $X$. (We make no claim that the conditions on $C(X)$ which determine whether or not $X$ is of second category are aesthetically pleasing or elegantly presented. For the record we give such criteria in 2.5 , but the merit of that section rests in its existence, not in its content.)

Since $G_{\delta}$-density is preserved by arbitrary products (in a sense

Presented to the Society, August 7, 1964; received by the editors July 6, 1964.

1 Each author received partial support from the National Science Foundation. 